\title{
Avulsions traumatiques des dents permanentes
}

\section{Traumatic avulsions of permanent teeth}

\section{MOTS-CLEFS :}

- Traumatisme dentaire, avulsion,

dent permanente,

réimplantation,

milieu de conservation,

endodontie,

résorption radiculaire

\section{KEYWORDS:}

- Dental trauma, avulsion permanent tooth, replantation, preservation medium, endotontics, root resorption

AOS 2013;266:4-13

DOI: $10.1051 / \mathrm{aos} / 2013602$

(C) EDP Sciences 2013

\section{Résumé}

L'avulsion dentaire traumatique constitue une urgence. En denture permanente, elle fait appel, le plus souvent, à la réimplantation. Aujourd'hui les conditions permettant le succès thérapeutique des réimplantations sont connues : il s'agit principalement des conditions de conservation et du temps extra-alvéolaire de la dent expulsée ainsi que des techniques de réimplantation et de contention qui lui sont appliquées. Cet article a pour objectif de faire le point sur les données actuelles de la littérature concernant le traitement des dents permanentes avulsées, la gestion des complications associées à ce traumatisme, ainsi que les moyens permettant de les prévenir ou d'en limiter les séquelles.

\section{Abstract}

\begin{abstract}
Traumatic dental avulsion is an emergency. In permanent dentures, it more often requires replantation. Today's conditions enabling successful replantation treatment are well known, and include mainly preservation and extra-alveolar time of the avulsed tooth and replantation and splint techniques. The aim of this article is to review the current literature data concerning the treatment of avulsed permanent teeth, the management of complications associated with this trauma, and the means to prevent or limit sequelae.
\end{abstract}

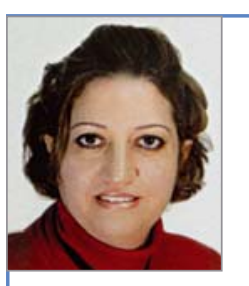

- S. ZOUITEN SKHIRI, Professeur en odontologie conservatrice, service de médecine dentaire, EPS Farhat Hached Sousse (Tunisie).

E. ABDELMOUMEN, Résidente en odontologie conservatrice, service de médecine dentaire, faculté de médecine dentaire de Monastir (Tunisie).

M. JEMAA, Résidente en odontologie conservatrice, service de médecine dentaire, faculté de médecine dentaire de Monastir (Tunisie).

N. DOUKI, Professeur en odontologie conservatrice, service de médecine dentaire, EPS Sahloul Sousse (Tunisie).

A. OUESLATI, Assistante en odontologie conservatrice, service de médecine dentaire, faculté de médecine dentaire de Monastir (Tunisie).

N. ZOKKAR, Professeur agrégé en odontologie conservatrice, service de médecine dentaire, faculté de médecine dentaire de Monastir (Tunisie).

N. CHAFRA, Technicienne supérieure, service de médecine dentaire, EPS Farhat Hached Sousse (Tunisie).

A. BOUGZALLAH, Professeur en orthodontie, service de médecine dentaire, EPS Farhat Hached Sousse (Tunisie). 


\section{INTRODUCTION}

Lavulsion dentaire traumatique correspond au déplacement complet de la dent hors de son alvéole.

Ces traumatismes concernent $8,7 \%$ à $30 \%$ de l'ensemble des accidents dentaires.

L'expulsion est beaucoup plus fréquente sur les dents permanentes immatures du fait de leurs racines plus courtes et de la laxité ligamentaire plus élevée [23-24].

Le traitement des dents permanentes avulsées est la réimplantation, immédiatement ou le plus rapidement après expulsion. La cicatrisation dépend presque entièrement de la période et de la manipulation extra-alvéolaire. Désormais, les complications pulpaires (nécroses) et parodontales (résorptions) sont très fréquentes après réimplantation [9-24]. D’après les données publiées, nous tenterons de définir un protocole thérapeutique adapté aux différentes situations d’avulsions traumatiques. Cette revue de littérature portera également sur les complications observées après réimplantation et les moyens permettant de les prévenir ou de limiter les séquelles.

\section{INDICATIONS ET CONTRE-INDICATIONS DES RÉIMPLANTATIONS}

Lavulsion dentaire traumatique constitue une urgence. Quand il s'agit d'incisives permanentes, l'indication est la réimplantation. Celle-ci devrait être tentée dans la majorité des cas, bien que noffrant parfois qu'une solution temporaire en raison de la fréquente survenue de résorption radiculaire. Néanmoins, même dans cette éventualité, la tentative de réimplantation se trouve largement justifiée : la dent réimplantée est susceptible de servir à maintenir l'espace idéal, et ceci pendant plusieurs années. Elle confère au patient une restitution ad integrum. En outre, en offrant un délai d'attente suffisant, elle permet au praticien d'évaluer l'importance à moyen terme des suites des traumatismes [2, 22, 25]. Cependant quelques situations contre-indiquent la réimplantation de la dent permanente dans le cas : Id'une pathologie générale, notamment pour les patients présentant un risque infectieux, des déficits immunitaires congénitaux ou acquis...;

Id'un gros fracas osseux ;

I d'un délabrement important de la dent avulsée (caries, fracture) $[3,8,30]$.

\section{PROTOCOLE DE RÉIMPLANTATION}

Le protocole comporte deux parties : la première consacrée au traitement d'urgence, la seconde au traitement différé. Le traitement d'urgence peut commencer avant même larrivée de l'enfant au cabinet dentaire par des conseils téléphoniques donnés aux parents ou aux personnes présentes au moment de laccident. Ces conseils portent principalement sur le mode de conservation de la dent expulsée. De nombreuses études ont montré linfluence néfaste du temps extra-alvéolaire sur le pronostic, surtout lorsque la dent est conservée à sec [5-8-31]. En effet, après 2 heures à sec, aucune cellule du ligament parodontal ne peut survivre [8-20]. Différents milieux ont été proposés pour garder la dent jusquà sa réimplantation. Les meilleurs seraient les milieux de culture cellulaire tels que le Viaspan ou la Hank's Balanced Salt Solution ${ }^{\circ}$ Un autre milieu, le Propolis ${ }^{\circ}$, proposé par Martin et Pileggi (2004), aurait des propriétés antibactériennes et anti-inflammatoires encore supérieures à celles des deux précédents $[8,19]$. Cependant, ces milieux sont peu disponibles en France et en Tunisie. Le lait serait un milieu correct, présentant une osmolarité et un PH favorables pour les cellules du ligament parodontal $[8,12,30]$. La conservation dans la salive est peu recommandée du fait de la septicité importante et de son caractère hypotonique. Il en est de même pour la conservation dans le vestibule buccal qui présente les mêmes inconvénients auxquels peuvent s'ajouter un problème psychologique et un risque d'ingestion. L'eau est très hypotonique et risque donc d'entraîner une lyse cellulaire. Cette solution ne doit donc être retenue que si aucun milieu n'est disponible $[8,26]$. Si la dent expulsée semble intacte (non souillée, non fracturée), l'idéal serait de la repositionner dans son alvéole sur le lieu même de laccident. Si cela nest pas possible, elle doit être conservée dans un milieu adéquat comme le lait ou, à défaut, dans du sérum physiologique et réimplantée dans les plus brefs délais $[8,21]$.

\section{Réimplantation immédiate}

(temps extra-alvéolaire < 60 minutes, milieu de conservation adéquat)

\section{Dent permanente immature (fig. 1)}

\section{- $1^{\text {re }}$ phase : traitement d'urgence}

„Prise de clichés radiographiques pour valider le bon état de l'alvéole.

I Maintenir la dent par la couronne et la nettoyer à l'aide d'un sérum physiologique. Surtout, ne pas gratter le ligament parodontal et ne pas assécher la racine avec un jet d'air ; pour favoriser la revascularisation la dent immature peut-être immergée pendant 5 minutes dans une solution de doxycycline (1 $\mathrm{mg}$ dilué dans $20 \mathrm{~mL}$ de sérum physiologique) [4-11].

I Administrer l’anesthésie locale.

„Débrider les tissus mous affectés. 


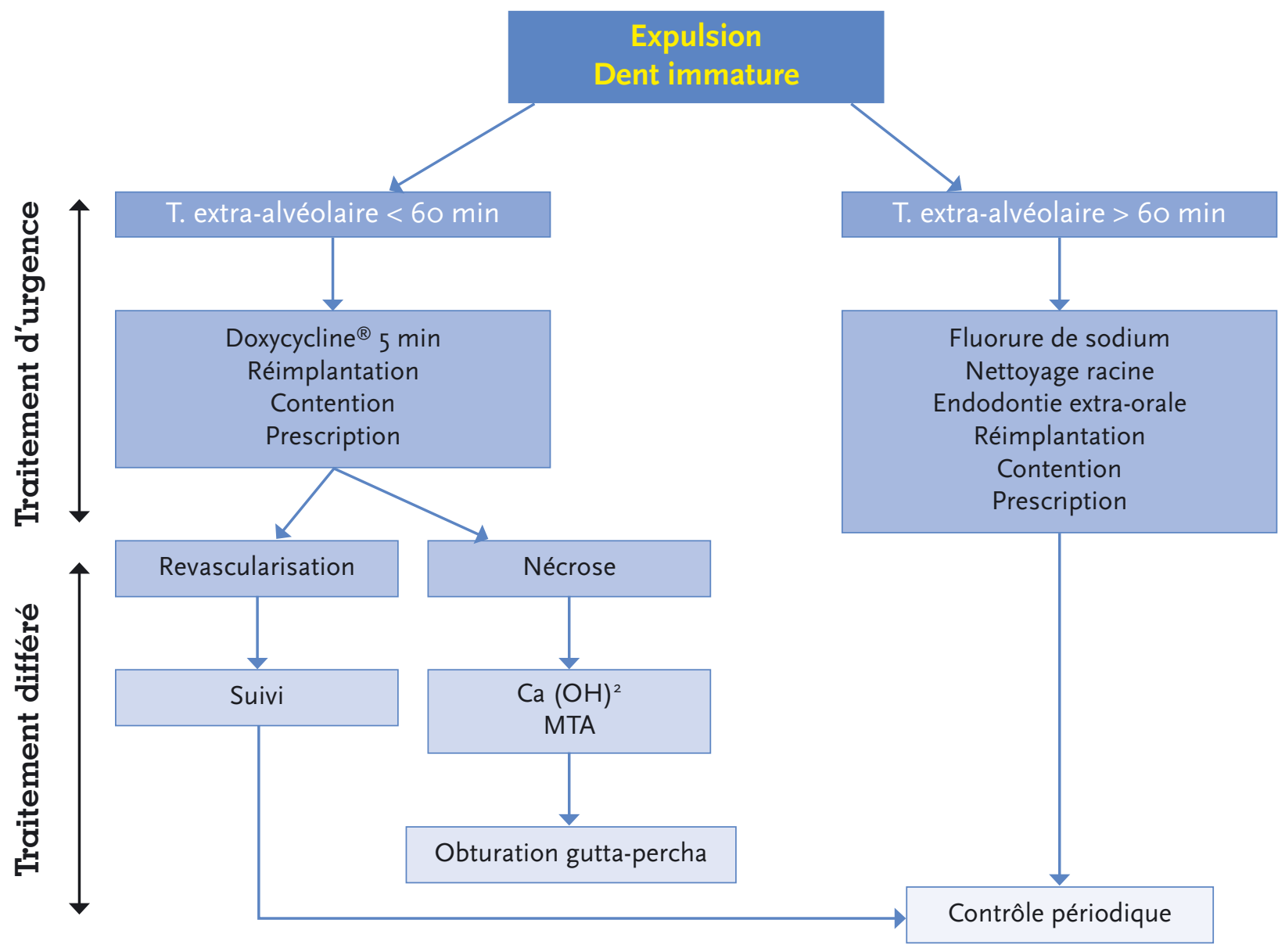

$\triangle$ Fig. 1 :

Arbre décisionnel des traitements d'urgence et différé des dents permanentes immatures après expulsion traumatique [9, 24].

| Irriguer l'alvéole à l'aide d'une solution saline pour éliminer le sang coagulé sans cureter l'alvéole.

„Réimplanter la dent avec une légère pression digitale sans pour autant forcer la dent en place.

I Vérifier l'occlusion pour s'assurer de l'absence d'interférence.

Immobiliser la dent pour une durée de sept à dix jours, à l'aide d'une attelle semi-rigide.

ISuturer les tissus mous, si nécessaire.

IPrendre des radiographies pour s'assurer du bon repositionnement de la dent dans son alvéole.

IExpliquer au patient l'importance d'une bonne hygiène buccodentaire et la nécessité d'effectuer des bains de bouche à la chlorhexidine.

„Prescrire un antibiotique et évaluer le besoin d'une toxine antitétanique [11].

\section{- $2^{e}$ phase : traitement différé}

Une revascularisation et une réinnervation pulpaire sont possibles. Dans ce cas, il y a lieu d'effectuer des contrôles cliniques et radiologiques. La vitalité pulpaire est testée régulièrement tous les mois. Cependant, des signes cliniques évidents de reprise de la vitalité ne pourront apparaître que deux mois plus tard. Le traitement d'apexification à l'hydroxyde de calcium ou au MTA (Mineral Trioxyde Aggregate) n’est instauré qu’en cas d'apparition d'une pathologie à la radiographie (résorptions ou lésion apicale) [24].

\section{Dent permanente mature (fig. 2)}

- $1^{\text {re }}$ phase : traitement d'urgence

Identique à la dent immature.

\section{- $2^{e}$ phase : traitement différé (traitement endodontique)}

Pour les dents à apex fermé, on procède au traitement endodontique le plus tôt possible.

Une semaine plus tard, le patient est revu et le tissu pulpaire est extirpé. La nécrose pulpaire peut ainsi être anticipée et les produits de dégradation pulpaire, générateurs de résorption inflammatoire, éliminés. Le parage canalaire s'effectue avec de l'hypochlorite à $2,5 \%$ et une obturation canalaire intermédiaire avec de l'hydroxyde de calcium est réalisée. Le traitement endodontique définitif à la gutta-percha peut être réalisé 6 à 12 mois après [24]. 


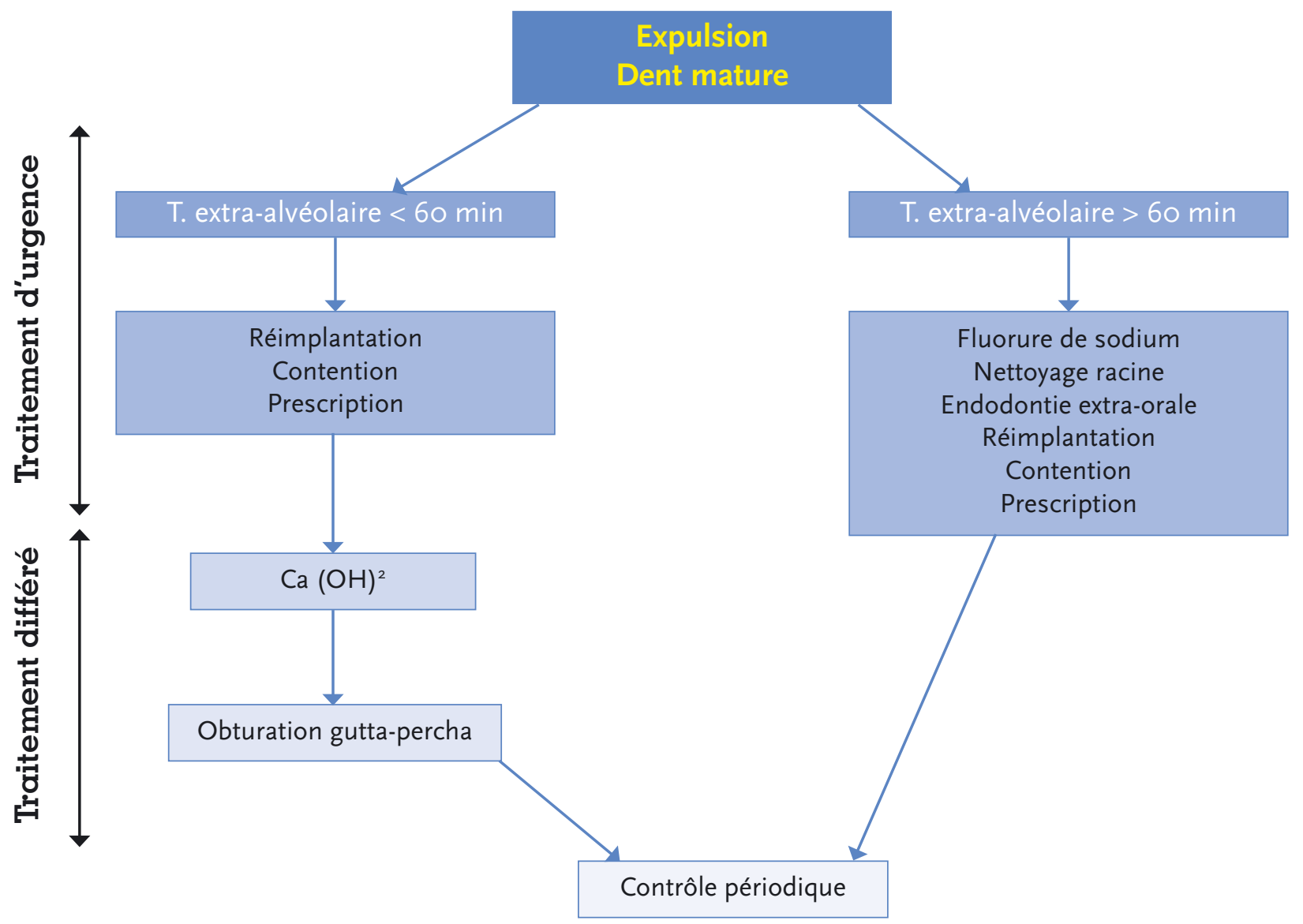

$\triangle$ Fig. 2 :

Arbre décisionnel des traitements d'urgence et différé des dents permanentes matures après expulsion traumatique [9-24].

\section{Réimplantation retardée (temps extra-alvéolaire > 60 minutes, milieu de conservation sec)}

\section{Dent permanente immature (fig. 1)}

Les opinions concernant la réimplantation retardée d'une dent immature avulsée divergent considérablement [11]. Nous préconisons l'approche de Trope [29], Flores et al. [14] qui recommandent de procéder selon le protocole de la réimplantation retardée d'une dent mature avulsée.

\section{Dent permanente mature (fig. 2)}

I Prise de clichés radiographiques pour valider le bon état de l'alvéole.

ı Éliminer le ligament parodontal nécrosé à l'aide de gazes.

IExtirper la pulpe.

I Immerger la dent dans une solution de fluorure de sodium $\mathrm{NaF}$ à $2 \%(\mathrm{pH}=5,5)$ pendant 20 minutes ou une solution de fluorure (APF à 1,23\%) pendant 15 minutes, afin d'incorporer des ions $\mathrm{F}$ aux surfaces cémentaires et dentinaires dans le but de retarder le processus de résorption [11, 22, 24, 32].
\Rincer la dent durant deux minutes à l'aide d'une solution saline.

„Procéder au traitement endodontique extra-oral et à lobturation du canal à la gutta-percha.

\Administrer l'anesthésie locale.

„Débrider les tissus mous affectés.

, Cureter et irriguer l'alvéole.

\Réimplanter la dent en exerçant une légère pression digitale sans pour autant forcer la dent en place.

IVérifier l'occlusion pour s'assurer de l'absence d'interférence.

\Immobiliser la dent pour une durée de six semaines au moyen d'une contention semi-rigide.

ISuturer les tissus mous, si nécessaire.

„Prendre des radiographies pour s'assurer du bon repositionnement de la dent dans son alvéole.

IExpliquer au patient l'importance d'une bonne hygiène buccodentaire et la nécessité d'effectuer des bains de bouche à la chlorhexidine.

„Prescrire un antibiotique et évaluer le besoin d'une toxine antitétanique.

Le suivi s'effectue de sept à dix jours après le traumatisme, de deux à trois semaines, de six à huit semaines, à six mois, à un an, puis annuellement pour les cinq années suivantes [11]. 


\section{COMPLICATIONS}

Des études sur lanimal ont montré que dans des conditions idéales de réimplantation, une guérison complète des tissus dentaires et de soutien peut être espérée [3-9]. Cependant, ces conditions idéales sont rarement réunies et les complications sont fréquentes. Les complications seraient plus importantes pour les dents immatures que pour les dents matures. La raison principale de la perte à long terme des dents réimplantées est la résorption radiculaire [7-9].

Différents types de résorptions externes peuvent être observés après réimplantation :

Iles résorptions de surface qui correspondent à des zones radioclaires limitées au niveau de la surface radiculaire et dont la guérison est spontanée et le pronostic favorable;

\les résorptions inflammatoires, liées au phénomène de nécrose pulpaire, qui se traduisent par des pertes de substance radiculaire ;

Iles résorptions de remplacement qui se traduisent par une fusion entre los alvéolaire et la surface radiculaire. La perte de substance causée par la résorption est comblée par du tissu osseux $[9,28]$.

\section{Quelle est la prévalence des résorptions?}

Les études cliniques montrent une prévalence des résorptions radiculaires variant de $50 \%$ à $100 \%$ selon les conditions de réimplantations [9]. Majorana et al., dans une étude portant sur 73 dents permanentes avulsées chez des patients âgés de 12 à 21 ans, ont constaté le développement de résorptions radiculaires dans $49,3 \%$ des cas [9, 17]. Boyd et al. ont publié une étude sur 50 dents avulsées dont les résultats montraient la présence de résorptions radiculaires après réimplantation dans $64 \%$ des cas. Dans $30 \%$ des cas, il s'agissait de résorptions inflammatoires, dans $52 \%$ des cas de résorptions de remplacement et, dans $18 \%$ des cas, les deux types de résorptions étaient présents [9-10].

\section{Quels facteurs influencent le développement des résorptions?}

Différents facteurs ont été mis en relation avec le développement des résorptions radiculaires après réimplantation.

\Le temps extra-alvéolaire: c'est semble-t-il le facteur le plus déterminant dans l'apparition du phénomène de résorption de remplacement ou ankylose : après 1 heure de conservation à sec, aucune survie pulpaire n'est possible; les cellules parodontales nont aucune chance de survie après 2 heures de conservation à sec $[1,23]$.
\Le degré de la contamination.

1Le nettoyage de la dent.

ILe délai avant réalisation de louverture de la chambre pulpaire.

ILa durée de la contention [9].

Une étude portant sur 128 dents réimplantées suivie pendant au moins deux ans a montré une relation significative entre développement de résorptions radiculaires et temps extra-alvéolaire à sec ainsi qu'entre résorptions radiculaires, contamination et traitement de la dent $[9,16]$ (tableau I).

Dans une autre étude, Kinirons et al. ont montré que le risque de résorption de remplacement augmentait significativement lorsque la durée de la contention était supérieure à 11 jours $[9,15]$ (tableau II). En revanche, en ce qui concerne le délai avant ouverture de la chambre pulpaire, il ne jouerait pas un rôle significatif par rapport aux résorptions [9] (tableau III).

\section{Quelle attitude adopter en présence de résorptions inflammatoires?}

La progression des résorptions inflammatoires est très rapide chez les patients jeunes [9-10]. Lattitude thérapeutique consiste en une désinfection endodontique et en une obturation à l'hydroxyde de calcium au long cours. Ce traitement permet un arrêt de la résorption et une stabilisation des lésions dans $50 \%$ des cas. Toutefois, le pronostic dépend beaucoup de la progression de la résorption avant l'intervention et de la mise en place de l'hydroxyde de calcium [9-17].

\section{Quelle attitude adopter en présence de résorptions de remplacement?}

Différents auteurs ont proposé de traiter les résorptions de remplacement avec de l'Emdogain ${ }^{\mathrm{TM}}$ (Straumann) qui est un produit composé de protéines matricielles amélaires hydrophobes extraites à partir d'émail porcin embryonnaire commercialisé depuis 1992 [8]. La technique décrite consiste à extraire la dent, à éliminer les tissus atteints par l'ankylose et à traiter la racine et l'alvéole à l'Emdogain ${ }^{\mathrm{TM}}$ avant réimplantation [9]. Les résultats d'une étude clinique réalisée en 2002 sur 16 dents avec un suivi moyen de 16 mois étaient encourageants puisque 11 dents sur 16 ne montraient aucun signe d'ankylose [13]. Cependant, plus récemment (en 2005), une nouvelle étude clinique portant sur 11 dents a montré des taux d'ankylose de $36 \%$ à 1 mois, de $72 \%$ à 2 mois et de $100 \%$ à 4 mois [27]. Ainsi, à ce jour, il nexiste aucun traitement satisfaisant de ces résorptions de remplacement. Souvent les résorptions de remplacement sont associées à une ankylose de la dent. Celle-ci correspond à un arrêt local 


\begin{tabular}{|c|c|c|c|}
\hline Temps de conservation à sec (secondes) & Résorption (\%) & Contamination et traitement de la dent & Résorption en (\%) \\
\hline $0-5$ & 51,4 & Nuls & 57,1 \\
\hline $6-20$ & 76,9 & Racine rincée & 75,0 \\
\hline \multirow{2}{*}{$21-180$} & \multirow{2}{*}{80,0} & Racine frottée & 87,5 \\
\cline { 2 - 4 } & & Contamination mais pas de nettoyage & 100,0 \\
\hline
\end{tabular}

$\triangle$ Tableau I [9-16]

\begin{tabular}{|c|c|}
\hline Durée de contention (jours) & Résorption de remplacement (\%) \\
\hline $4-10$ & 16,7 \\
\hline $11-19$ & 57,6 \\
\hline$>20$ & 59,3 \\
\hline
\end{tabular}

$\triangle$ Tableau II [9-15]

\begin{tabular}{|c|c|}
\hline Délai avant traitement endodontique (jours) & Résorption (\%) \\
\hline 0-10 & 25,0 \\
\hline $11-19$ & 25,0 \\
\hline$>20$ & 39,1 \\
\hline Pas de traitement & 11,1 \\
\hline
\end{tabular}

$\triangle$ Tableau III [9-15]

de la croissance alvéolaire. Chez un enfant jeune dont la croissance n'est pas terminée, l'ankylose va entraîner une infraposition de la dent atteinte et une version des dents adjacentes. L'extraction est donc nécessaire avant que ces modifications ne deviennent trop importantes et compromettantes pour une thérapeutique prothétique future [9].

Les conséquences néfastes apparaissent lorsque l'infraclusion a progressé jusquà la moitié de la hauteur coronaire de la dent adjacente. Certains auteurs considèrent même que l'extraction ou la décoronation est indiquée dès que l'infraposition est modérée (entre $1 / 8$ et $1 / 4$ de la hauteur coronaire) $[9,18]$. Toutefois, le maintien de la dent sur l'arcade permet la conservation de l'espace et de l'esthétique $[6,9]$. Notons enfin que la progression de l'infraclusion varie individuellement et en fonction de la croissance du patient. Si l'ankylose est diagnostiquée avant lâge de 10 ans, le risque d'infrapo- sition sévère est très élevé. Ce processus s'accroît de manière considérable au moment de la puberté quand la croissance faciale est très rapide $[9,18]$.

\section{CONCLUSION}

Les complications sont fréquentes après réimplantation, particulièrement pour les dents permanentes immatures. Toutefois la réimplantation devrait toujours être tentée en l'absence de contre-indication. Même si elle n'offre parfois qu'une solution temporaire en raison de la fréquente survenue de complications, la tentative de réimplantation se trouve largement justifiée : la dent réimplantée est susceptible de servir de mainteneur d'espace idéal et ceci pendant plusieurs années : elle confère au patient une restitution ad integrum en attendant une solution définitive $[9,25]$.

Cas cliniques

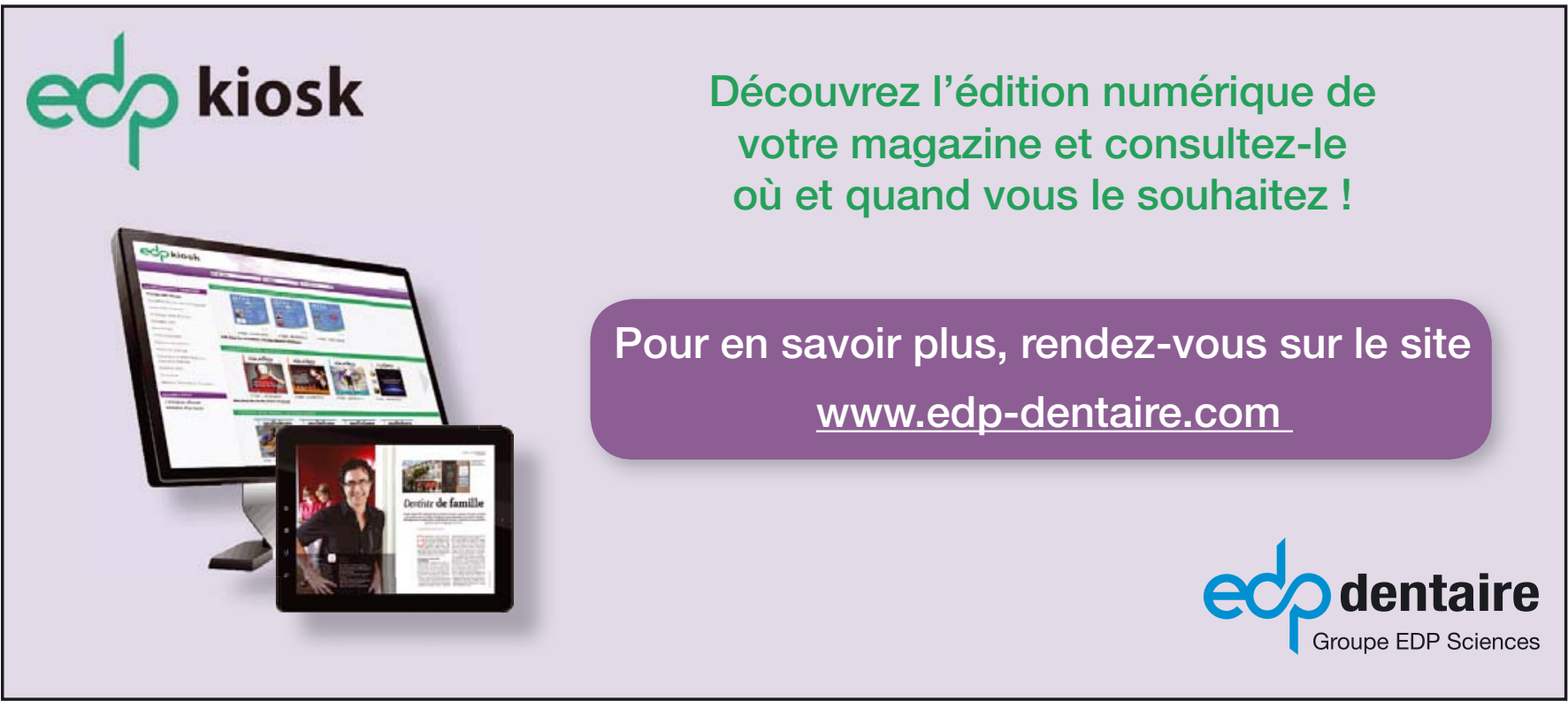




\section{CAS CLINIQUES}

\section{Cas clinique $n^{\circ} 1$}

Une patiente âgée de 8 ans s'est présentée à peu près 1 heure après un accident de la voie publique ayant entraîné l'expulsion de la 11, la luxation partielle de la 12 , ainsi que des subluxations au niveau de la 13, 21, 22, 23. La dent expulsée conservée au sec (dans une serviette de papier) a été réimplantée et immobilisée. Une contention à l'aide de bracket orthodontique réalisée par un spécialiste est mise en place pendant 15 jours (fig. 3). Un contrôle périodique a été instauré et les dents ne présentaient aucun signe pathologique, seulement à 3 mois la 11 présentait une nécrose pulpaire. Un traitement endodontique à la gutta a été réalisé après quatre séances d'hydroxyde de calcium. Puis la patiente est perdue de vue. Onze ans plus tard, elle s'est présentée de nouveau à la consultation, se plaignant de gêne au niveau des dents de sagesse supérieures. L'examen clinique a montré :

rau niveau de la 11 : une dent immobile toujours présente sur l'arcade, légèrement en infraclusion, présentant une déformation de l'architecture gingivale (fig. 4); I au niveau de la $13,21,22,23$, les dents sont vivantes sans aucune mobilité anormale; en revanche, la 12

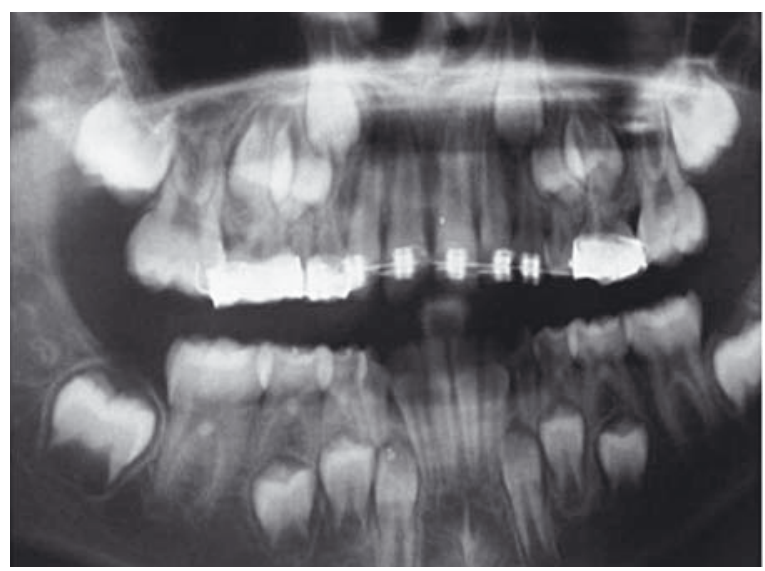

$\triangle$ Fig. 3 :

Radio panoramique 4 jours après réimplantation, contention en place. répond négativement aux tests de vitalités pulpaires et présente une légère douleur à la percussion axiale; $\mathrm{du}$ côté palatin, on note la présence de ciment verre ionomère obturant la cavité d'accès (d'après l'anamnèse, une tentative de traitement endodontique au niveau de la 12 a été instaurée par un médecin de sa région). L'examen radiologique (fig. 5a, 5b) a mis en évidence une série de séquelles :

I au niveau de la 11, deux types de résorptions :

- une résorption de remplacement presque complète de la racine, la gutta-percha obturant le canal a été parfaitement respectée par le processus de résorption et sa présence est visible sur le cliché ;

- une résorption inflammatoire caractérisée par des zones radio claires;

rau niveau de la 12 : on note la présence d'une image périapicale témoignant de la mortification pulpaire et confirmant les tests cliniques ainsi qu'une réduction du volume pulpaire (signe de revascularisation pulpaire dans le temps).

Cette patiente retrouve toujours son sourire et refuse complètement l'extraction de la 11 et le traitement de la 12.

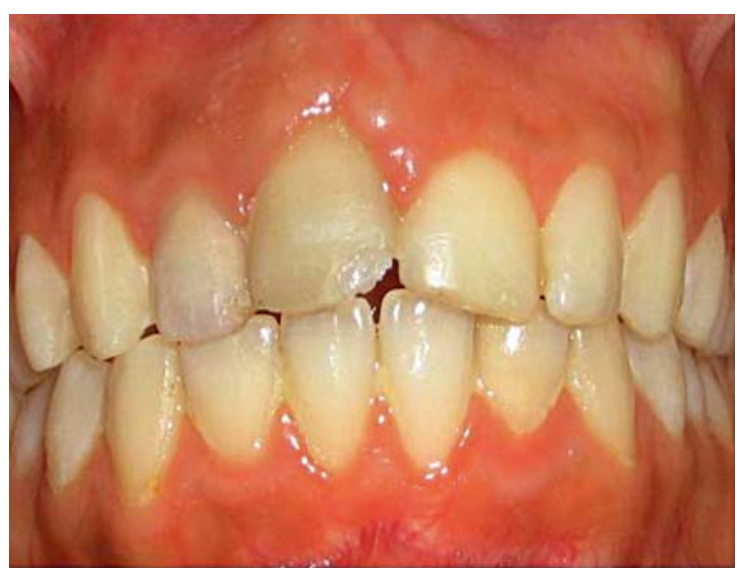

$\triangle$ Fig. 4 :

Vue clinique 11 ans après réimplantation. 11 toujours en place.
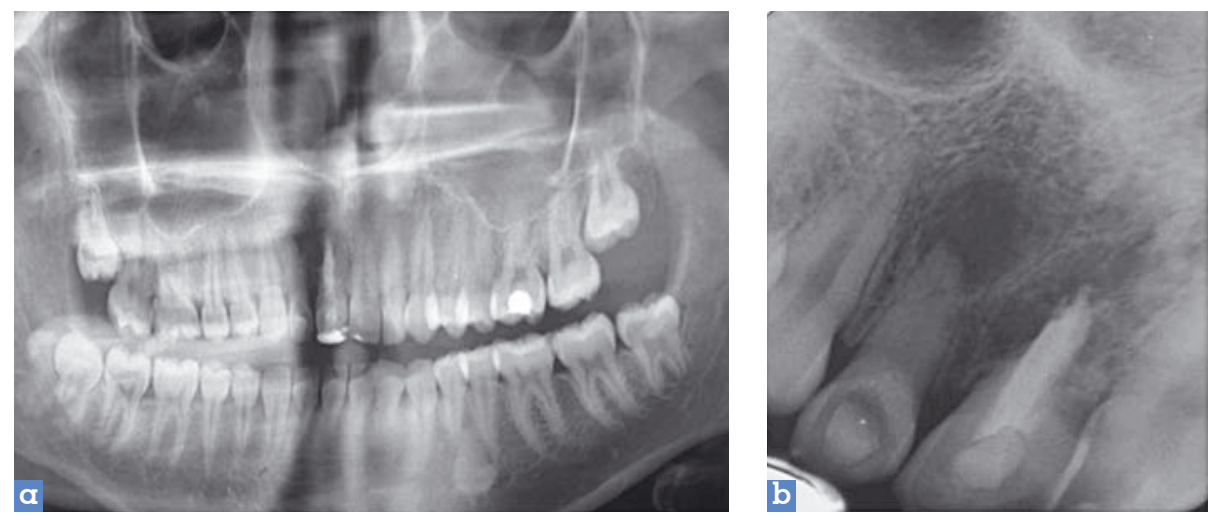

$\triangleleft$ Fig. 5 :

Radio 11 après réimplantation

a. Radio panoramique

b. Radio rétroalvéolaire :

- 11 résorption radiculaire de remplacement et inflammatoire. Noter la persistance de gutta au sein de l'os alvéolaire

- 12 : Présence d'une image périapicale. Noter la réduction du volume pulpaire. 


\section{Cas clinique $\mathrm{n}^{\circ} \mathbf{2}$}

Un patient âgé de 13 ans s'est présenté à la consultation 40 minutes après l'accident, présentant dans un mouchoir la 11 expulsée. La dent a été réimplantée et immobilisée.

Après une semaine, le tissu pulpaire est extirpé et le canal obturé à l'hydroxyde de calcium. Le traitement endodontique définitif à la gutta est réalisé 6 mois après, sachant que l'hydroxyde de calcium a été renouvelé tous les 2 mois. Trois ans plus tard, le patient s'est présenté de nouveau à la consultation se plaignant de gêne au niveau de la 11 .

\L'examen clinique de la 11 a révélé la présence de coloration bleuâtre, dent immobile, en infraposition indiquant avec certitude une ankylose (fig. 6a). Une cavité d'accès ouverte (fig. 6b) présentant une odeur fétide, ce qui a conduit d'ailleurs le patient à consulter.

\L'examen radio de la 11 a objectivé une résorption radiculaire inflammatoire sévère ainsi que des lacunes de résorption caractéristiques déclenchées par une infection (fig. 7). La résorption avait tellement progressé que l'extraction s'est avérée nécessaire. La dent extraite a été récupérée (fig. $\mathbf{8 a}, \mathbf{8 b}$ ) et une prothèse amovible a été réalisée (fig. 9) jusqu'a la réalisation d'un bridge collé qui est une solution préférable chez les adolescents.

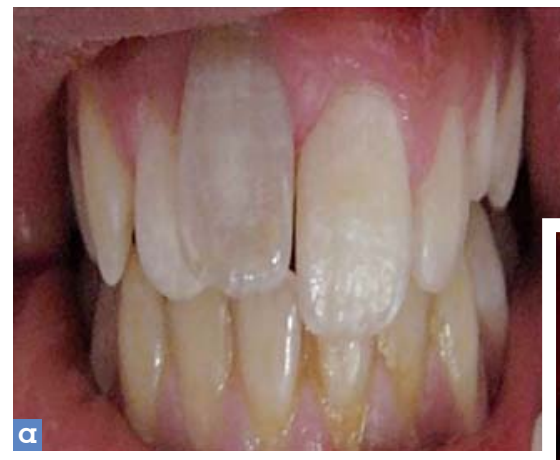

$\triangleleft$ Fig. 6 :

Vue clinique de la 11, 3 ans après réimplantation.

a. Vue vestibulaire: 11 de coloration grisâtre en infraclusion

b. Vue palatine : - Cavité d'accès ouverte infectée - Gencive hypertrophiée.

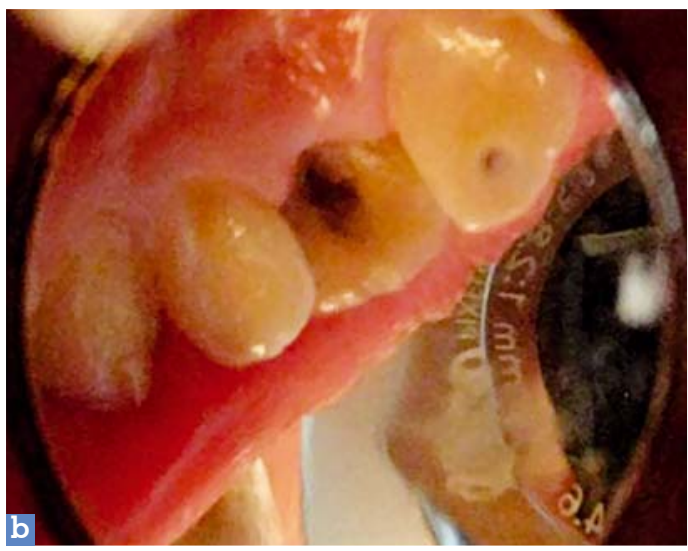

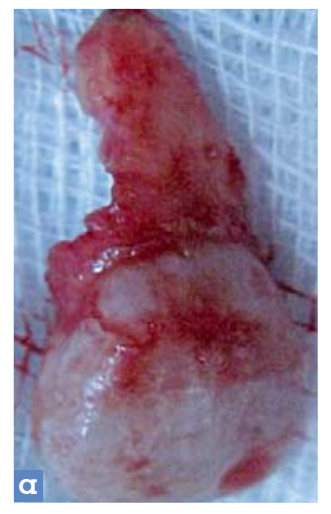

Fig. 8 : Dent extraite récupérée fortement résorbée a. Vue vestibulaire, b. Vue de profil.

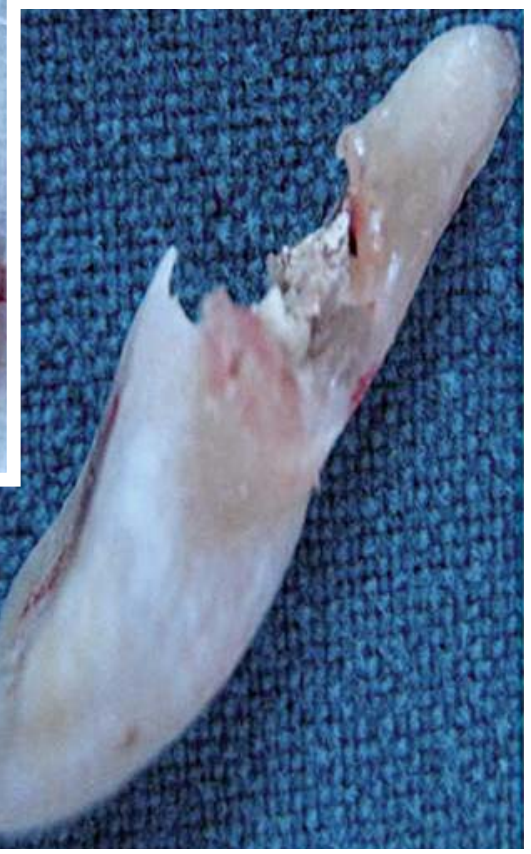

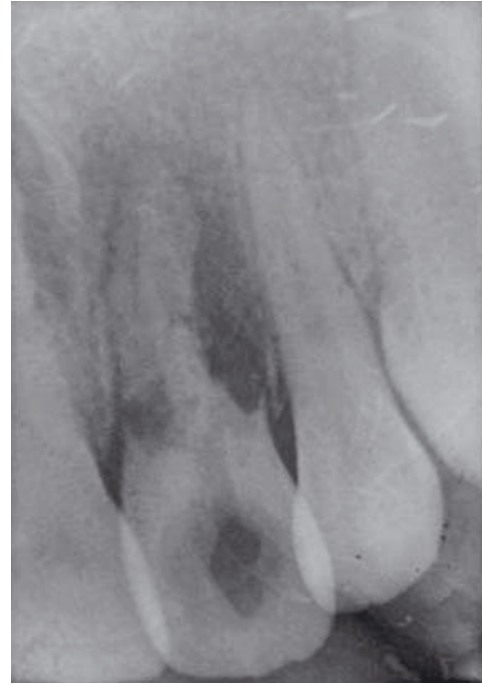

$\triangle$ Fig. 7 :

Radio 3 ans après réimplantation.

- Résorption radiculaire externe sévère

- Tissu dentaire résorbé remplacé par du tissu de granulation (radio claire).

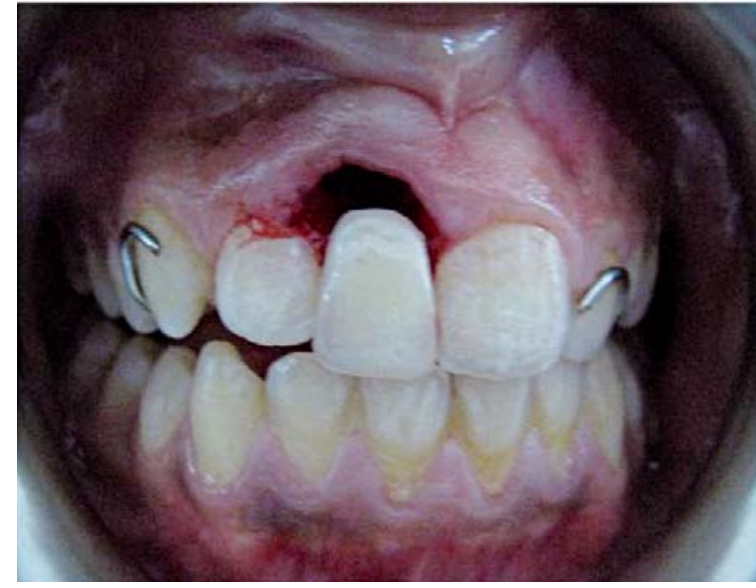

$\triangle$ Fig. 9 :

Prothèse immédiate amovible en place. 

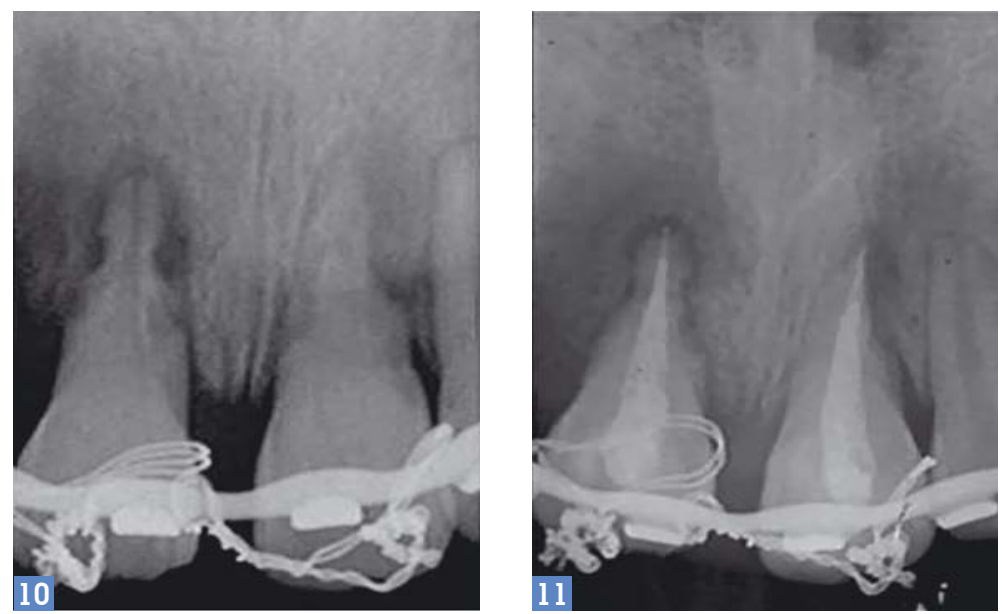

$\triangle$ Fig. 10 :

1 mois après réimplantation. Noter la présence de résorptions radiculaires inflammatoires au niveau de la 11 et 21 réimplantées.

$\triangle$ Fig. 11 :

- 11, 21 obturées à la Gutta après 3 séances d'hydroxyde de calcium - la mise en place d'hydroxyde de calcium a permis de ralentir la progression du Phénomène de résorption.

\section{Cas clinique $n^{\circ} 3$}

Une patiente âgée de 14 ans s'est présentée à la consultation une heure après l'accident, présentant la 11 et la 21 expulsées dans un mouchoir en papier. La réimplantation et l'immobilisation ont été effectuées. Malheureusement, la patiente est revenue 4 semaines après, bien qu'elle ait été informée au préalable des risques. Au niveau de la 11 et 21, l'examen clinique a révélé des dents mobiles dyschromiées.

Lexamen radiologique (fig. 10) a dévoilé la présence de résorptions inflammatoires marquées. Probablement cette résorption n'aurait pas lieu si la patiente avait été revue 7 jours après la réimplantation pour la réalisation du traitement endodontique. Le traitement endodontique définitif à la gutta a été effectué après 3 séances d'hydroxyde de calcium (fig. 11). Le suivi après 5 mois a révélé des dents très mobiles, douloureuses à la mastication. L'absence de motivation et de coopération de la patiente nous a poussés à entamer l'extraction des dents.

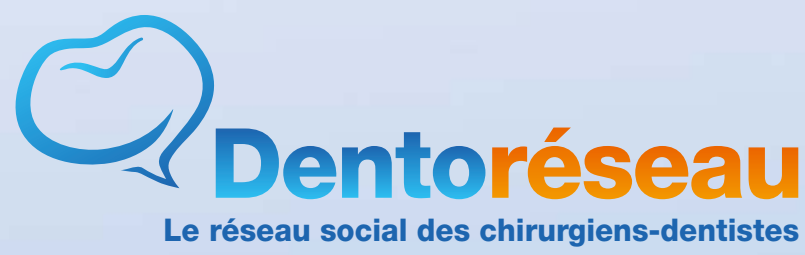

\section{Rejoignez le réseau social des chirurgiens-dentistes}

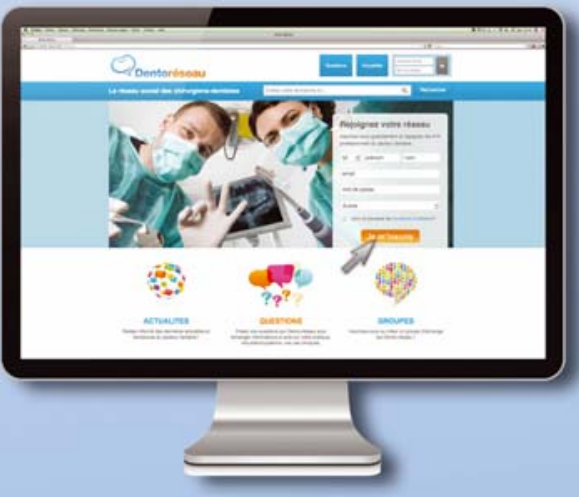

2 Découvrir et rejoindre une communauté de confrères

$\mathcal{Q}$ Participer et animer des groupes de discussion

Partager et actualiser ses connaissances

Créer et enrichir son réseau

\section{www.dento-reseau.com}

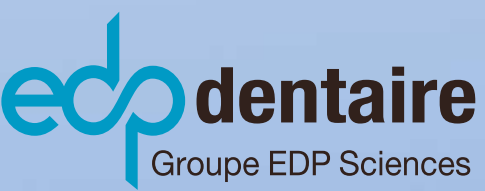




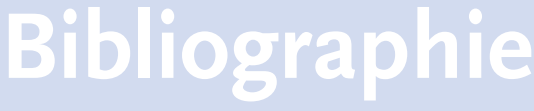

[1] Andreasen JO. Effect of pulp extirpation or root canal treatment on periodontal healing after replantation of mature permanent incisors in monkeys. J Endod 1981;7:245-252.

[2] Andreasen JO. Exarticulations. In: Traumatic injuries of the teeth, $2^{\text {nd }}$ ed. Munksgaard, Copenhagen \& Saunders. Philadelphie, 1981:203-242.

[3] Andreasen JO, Andreasen FM. Textbook and color atlas of traumatic injuries to the teeth. Copenhagen, Munsgaard,1994.

[4] Andreasen JO., Andreasen FM. Avulsions. In: Andreasen JO, Andreasen FM, Andersson L. Textbook and Color Atlas of Traumatic Injuries to the Teeth. 4th ed. Copenhagen : Blackwell Munksgaard, 2007:444-88.

[5] Andreasen JO, Borum MK, Jacobsen HL, Andreasen FM. Replantation of 400 avulsed permanent incisors. Endod Dent Traumatol 1995;11:51-89.

[6] Australas AR. The avulsed tooth: the best implant. Coll Dent Surg 2000;15:243-246.

[7] Barrett EJ, Kenny DJ. Survival of avulsed permanent maxillary incisors in children following delayed replantation. Endod Dent Traumatol 1997;13:269-275.

[8] Beslot-Neveu A, David-Béal T, Villette F. Avulsion traumatique des dents permanentes : Gestion de la consultation d'urgence. Clinic 2009;30:253-259.

[9] Beslot-Neveu A, David-Béal T,Villette F. Avulsions traumatiques des dents permanentes : Traitement différé et gestion des complications. Clinic Juin 2009;30:303-308.

[10] Boyd DH, Kinirons MJ, Gregg TA.

A prospective study of factors affecting survival of replanted permanent incisors in children. Int J Ped Dent 2000;10:200-205.
[11] Charland R, et al. Traumatismes des dents antérieures permanentes. Treizième partie : avulsion. Journal de l'Ordre des dentistes du Québec 2008;45:63-69.

[12] Doyle DL, Dumsha TC, Sydiskis RJ. Effect of soaking in Hank's balanced salt solution or milk on PDL cell viability of dry stored human teeth. Endod Dent Traumatol 1998;14;221-224.

[13] Flippi A, Pohl Y, Von Arx T. Treatment of replacement with Emdogain: a prospective clinical study. Dent traumatol 2002;18:138-143.

[14] Flores MT, et al. Guidelines for the management of traumatic dental injuries. II. Avulsion of permanent teeth. Dent Traumatol 2007;23:130-6.

[15] Kinirons MJ, Boyd DH, Gregg TA. Inflammatory and replacement resorption in reimplanted permanent incisor teeth : a study of the characteristics of 84 teeth. Endod Dent Traumatol 1999;15:269-272.

[16] Kinirons MJ, Gregg TA, Welbury RR, Cole $B$. Variations in the presenting and treatment features in reimplanted permanent incisors in children and their effect on the prevalence of root resorption. Br Dent J 2000;189;263-265.

[17] Majorana A, Bardellini E, Conti G, Keller E, Pasini S. Root resorption in dental trauma: 45 cases followed for 5 years. Dent Traumatol 2003;19:262-265.

[18] Malmgren B, Malmgren O. Rate of infraposition of reimplanted ankylosed incisors related to age and grouth in children and adolescents. Dent Traumatol 2002;18:28-36.

[19] Martin MP, Pileggi R. A quantitative analysis of Propolis : a promising new storage media following avulsion. Dent Traumatol 2004;20:85-89.

[20] Martins WD, Portela V, Westphalen D, Westphalen FH. Tooth replantation after traumatic avulsion: a 27-year follow-up. Dent Traumatol 2004;20:101-105.
[21] Mcintyre JD, Lee JY , Trope M, Vann NF. Management of avulsed permanent incisors: a comprehensive update. Clinic mai 11. Pediatr Dent 2007;29:56-63.

[22] Mc Tigue DJ. Managing traumatic injuries in the yong permanent dentition. In: Pediatric Dentistry. Pinkham JR(ed), Saunders, Philadelpia, p 417-418, 1988.

[23] Naulin-IFI C. Polytraumatisme des dents et du parodonte. Encycl Méd Chir (Elseiver, Paris), Odontologie, 23-008-A-10,1998,12 p

[24] Naulin-IFI C. Traumatisme dentaire du diagnostic au traitement. Editions $C d P$, Groupe Liaisons, 2005.

[25] Obry F, Sommerrater J. Les expulsions dentaires. Réalités cliniques 1992;3 471-479.

[26] Ram D, Cohenca N. Therapeutic protocols for avulsed permanent teeth: review and clinical update. Pediatr Dent 2004;26:251-255.

[27] Schjott M, Andreasen JO. Emdogain does not prevent progressive root resorption after replantation of avulsed teeth: a clinical study. Dent Traumatol 2002;18:28-36.

[28] Tronstad L. Root resorption-etiology, terminology and cinical manifestations. Endod Dent Traumatol 1988;18:109-118.

[29] Trope M. Clinical management of the avulsed tooth: present strategies and future directions. Dent Traumatol 2002; 18:1-11.

[30] Trope M. Clinical management of the avulsed tooth. Dent Clin North Am 1995;39:93112.

[31] Trope M, Hupp JG, Mesaros SV. The role of the socket in the periodontal healing of replanted dog's teeth stored in Viaspan for extended periods. Endod Dent Traumatol 1997;13:171-175.

[32] Zuckerman LS, Fuss Z, Ashkenazi M. New emphasis in the treatment of dental trauma : avulsion and luxation. Dent Traumatol 2007;23:297-303.

\section{edpdentaire \\ La référence du monde dentaire}

ACtuAlitÉS

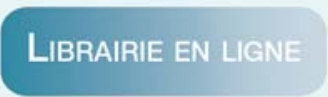

\section{EDP Dentaire vous propose I'intégralité de son offre sur www.edp-dentaire.fr}

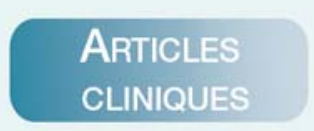

Prodults

Organisation DU CABINET

Protocoles

\section{Agenda}

